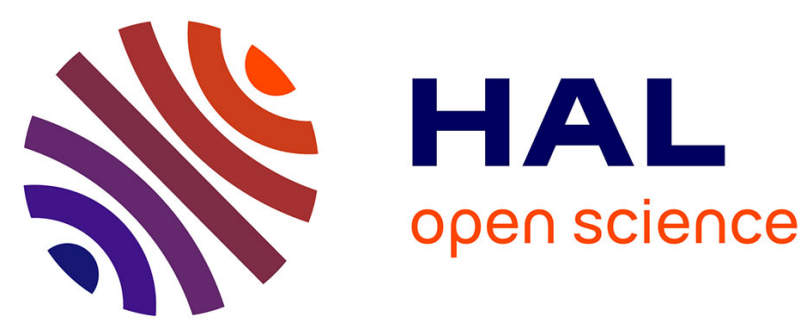

\title{
The Xenopus tadpole: an in vivo model to screen drugs favoring remyelination
}

\author{
Abdelkrim Mannioui, Quentin Vauzanges, Jean Baptiste Fini, Esther Henriet, \\ Somya Sekizar, Loris Azoyan, Jean Léon Thomas, David Du Pasquier, Carine \\ Giovannangeli, Barbara A. Demeneix, et al.
}

\section{To cite this version:}

Abdelkrim Mannioui, Quentin Vauzanges, Jean Baptiste Fini, Esther Henriet, Somya Sekizar, et al.. The Xenopus tadpole: an in vivo model to screen drugs favoring remyelination. Multiple Sclerosis Journal, 2018, 24 (11), pp.1421 - 1432. 10.1177/1352458517721355 . hal-01912256

\section{HAL Id: hal-01912256 https://hal.sorbonne-universite.fr/hal-01912256}

Submitted on 5 Nov 2018

HAL is a multi-disciplinary open access archive for the deposit and dissemination of scientific research documents, whether they are published or not. The documents may come from teaching and research institutions in France or abroad, or from public or private research centers.
L'archive ouverte pluridisciplinaire HAL, est destinée au dépôt et à la diffusion de documents scientifiques de niveau recherche, publiés ou non, émanant des établissements d'enseignement et de recherche français ou étrangers, des laboratoires publics ou privés. 


\section{The Xenopus tadpole: an in vivo model to screen drugs favoring remyelination}

Abdelkrim Mannioui, PhD, ${ }^{1}$ Quentin Vauzanges, MS, ${ }^{1}$ Jean Baptiste Fini, PhD,${ }^{2}$ Esther Henriet, MS, ${ }^{1}$ Somya Sekizar,PhD, ${ }^{1}$ Loris Azoyan, MS, ${ }^{1}$ Jean Léon Thomas, $\mathrm{PhD},{ }^{1,3}$, David Du Pasquier, $\mathrm{PhD},{ }^{4}$ Carine Giovannangeli, $\mathrm{PhD},{ }^{5}$ Barbara Demeneix, $\mathrm{PhD},{ }^{2}$ Catherine Lubetzki, $\mathrm{MD}, \mathrm{PhD},{ }^{1}$ and Bernard Zalc, MD, $\mathrm{PhD}^{1}$

${ }^{1}$ Sorbonne Universités UPMC Univ Paris 06, Inserm, CNRS, APHP, ICM-GH Pitié-Salpêtrière, F-75013 Paris, France ;

${ }^{2}$ CNRS UMR 7221, Muséum National d'Histoire Naturelle, F-75005 Paris, France;

${ }^{3}$ Department of Neurology, Yale University School of Medicine, New Haven, USA;

${ }^{4}$ Watchfrog, 91000 Evry, France;

${ }^{5}$ CNRS UMR 7196, Muséum National d’Histoire Naturelle, F-75005 Paris, France

Keywords: Multiple sclerosis, Xenopus laevis, transgenic, demyelination, remyelination, Crispr/Cas9, oligodendrocyte, gene editing.

Address correspondence to: Dr Bernard Zalc, Institut du Cerveau et de la Moelle épinière (ICM); GH Pitié-Salpêtrière - 47 boulevard de l’Hôpital, 75013 Paris, France Tel. +33 (0)1 572744 71- e.mail : bernard.zalc@upmc.fr

Number of characters in the title: 65

\section{Number of words:}

Abstract: 186

Body of the manuscript (Abstract, Introduction, M\&M, Results, Discussion): 3652

Number of figures: 5, Color figures: 4, Table: 1 (Supplementary files: 2 figures: 1 B\&W and 1 color) 


\section{Abstract}

Background: In MS development of screening tools for remyelination-promoting molecules is timely.

Objective: A Xenopus transgenic line allowing conditional ablation of myelinating oligodendrocytes has been adapted for in vivo screening of remyelination-favoring molecules.

Methods: In this transgenic, the green fluorescent protein reporter is fused to E. coli nitroreductase and expressed specifically in myelinating oligodendrocytes. Nitroreductase converts the innocuous pro-drug metronidazole to a cytotoxin. Spontaneous remyelination occurs after metronidazole-induced demyelinating responses. As tadpoles are transparent, these events can be monitored in vivo and quantified. At the end of metronidazole-induced demyelination, tadpoles were screened in water containing the compounds tested. After $72 \mathrm{~h}$ remyelination was assayed by counting numbers of oligodendrocytes per optic nerve.

Results: Among a battery of molecules tested, siponimod, a dual agonist of sphingosine-1phosphate receptor 1 and 5, was among the most efficient favoring remyelination. Crispr/cas9 gene editing showed that the promyelinating effect of siponimod involves the sphingosine-1phosphate receptor 5 .

Conclusion: This Xenopus transgenic line constitutes a simple in vivo screening platform for myelin repair therapeutics. We validated several known pro-myelinating compounds, and demonstrated that the strong remyelinating efficacy of siponimod implicates the sphingosine-1phosphate receptor 5 . 


\section{Introduction}

Oligodendrocytes are the myelin-forming cells of the CNS. During embryonic development, cells of the oligodendrocyte lineage emerge from the ventricular zone. Successive developmental stages transform the neural stem cells into proliferating oligodendrocyte progenitors, which migrate into the parenchyma as oligodendrocyte precursor cells (OPCs) before differentiating, successively into a pre-oligodendrocyte, then a mature postmitotic oligodendrocyte and finally a myelin-forming oligodendrocyte. These developmental stages, which are associated with different capacities of proliferation, migration, differentiation, survival, maturation, and finally myelin deposition, are cell autonomous, but also depend on a variety of extrinsic factors.

In multiple sclerosis (MS) remyelination often fails despite OPC presence around demyelinating areas. ${ }^{1}$ Repair of demyelinated lesions could enhance successful re-ensheathing of naked axons by oligodendrocyte processes. Existing MS models (mostly rodent and primates) are not ideal for monitoring in vivo demyelination and remyelination, hence the need to develop simpler strategies for larger scale screening of compounds favoring myelin repair. ${ }^{2}$ We developed a transgenic Xenopus laevis permitting live imaging of demyelination and remyelination by conditionally triggering apoptotic ablation of myelin forming oligodendrocytes. ${ }^{3}$ This line, MBP-GFP-NTR, expresses green fluorescent protein (GFP) fused to E. coli nitroreductase (NTR) under control of the mouse $1.9 \mathrm{~kb}$ myelin basic protein $(\mathrm{MBP})$ regulatory sequence. In the mouse, this portion of the proximal upstream region of $M B P$ gene drives reporter expression specifically in myelinating oligodendrocytes. ${ }^{4}$ Like the mouse, the transgenic MBP-GFP-NTR X. laevis expresses the GFPNTR transgene specifically in myelin-forming oligodendrocytes.

The NTR enzyme converts the nitro radical of prodrugs, including metronidazole, to a highly cytotoxic hydroxylamine derivative. In metronidazole-exposed transgenic MBP-GFP-NTR tadpoles demyelination is restricted to the CNS, without axonal damage. ${ }^{3}$ The extent of demyelination depends on the concentration and duration of metronidazole exposure. 
Spontaneous remyelination occurs and is nearly complete 8 days after cessation of metronidazole. As embryos are transparent, demyelination and remyelination can be monitored and quantified in vivo under a fluorescent macroscope. ${ }^{3}$ We used this model to screen molecules favoring remyelination. Among the battery of molecules tested, one of the most potent to accelerate remyelination was siponimod, which was recently evaluated in a Phase III trial in secondaryprogressive MS. ${ }^{5}$

Siponimod is a dual agonist of sphingosine-1-phosphate receptors, S1PR1 and S1PR5. Sphingosine-1-phosphate (S1P) has been identified as the endogenous ligand of the five known S1P receptors (S1PR1, S1PR2, S1PR3, S1PR4 and S1PR5) a family of G-protein coupled receptors. ${ }^{6}$ In the rodent CNS, the S1PR5 receptor is expressed only by cells of the oligodendroglial lineage and its activation is important in the oligodendrocyte maturation. It was therefore suggested that S1PR5 may play a key role in myelin sheath repair. ${ }^{7}$ Here we questioned whether the remyelinating effect of siponimod implicated S1PR5. We show that, in tadpoles with Crispr/Cas 9 gene deleted S1PR5, siponimod no longer promotes remyelination. Another S1PR5 agonist (UC-42-WP04) also favored remyelination, while an S1PR1 agonist (AUY954) had no effect. The data provide strong evidence that siponimod-stimulated remyelination implicates S1PR5 activation and illustrate that MBP-GFP-NTR Xenopus is an efficient and simple experimental model to screen molecules favoring remyelination. 


\section{Materials and methods}

\section{Animals}

Xenopus tadpoles were staged according to Nieuwkoop and Faber. ${ }^{8}$ X. laevis is an allotretraploid composed of two separate diploid genomes $(2 \mathrm{n}=36)$. X. laevis has nine pairs of chromosomes named Xla1S and Xla1L through Xla9S and Xla9L where S (short) and L (long) refer to their relative lengths. Tadpoles of either sex were anesthetized in 0.05\% MS-222 (Ethyl-3aminobenzoate methanesulfonate; Sigma-Aldrich) before quantification of GFP+ cells, and returned to normal water to recover. Before brain and spinal cord dissection, tadpoles were euthanized in $0.5 \%$ MS-222. Animal care was in accordance with institutional and national guidelines. All animal procedures conformed to the European Community Council 1986 directive (86/609/EEC) as modified in 2010 (2010/603/UE) and have been approved by the ethical committee of the French Ministry of Higher Education and Research. Transgenic tadpoles were treated between stages NF 50 and 55, corresponding to pre-metamorphosis, a stage of ongoing myelination.

\section{Antibodies}

Mouse anti-MBP (directed against Xenopus MBP, 1:1000, kindly provided by Dr. Saburo Nagata), chick anti GFP (1:1000, Aves Lab, USA), rabbit anti-S1PR5 antibody (1:100 Imgenex, USA), goat anti-human Sox10 (1:50 R\&D systems). Corresponding secondary antibodies conjugated Alexa 350, Alexa 488, Alexa 594 and Alexa 647 (1: 1000, Invitrogen). For CLARITY we used chicken anti-GFP, Avès Labs, 1:400 as primary antibody and goat anti-chicken Alexa Fluor 488, Invitrogen, 1:600 as secondary one.

CLARITY and Imaging using a high refractive index solution are detailed in the supplementary materials. 


\section{Immunostaining of clarified samples}

CLARITY-processed brains were incubated in blocking solution overnight at $4^{\circ} \mathrm{C}$. Samples were further incubated in staining solution with primary antibodies (anti-GFP) for $7 d$ at room temperature with under gentle agitation. After 2 washes with PBT, samples were incubated in a staining solution with secondary antibody for $7 d$ at room temperature. Samples were subsequently washed for $48 \mathrm{~h}$ in PBT.

\section{Immunolabeling on tissue sections}

Tadpoles were fixed by immersion in $4 \%$ paraformaldehyde and treated for immunostaining as described. $^{3}$

Electron microscopy and morphometry are detailed in the supplementary materials.

\section{Metronidazole preparation and use}

Metronidazole (10mM, Fluka) was dissolved in filtered tap water containing 0.1\% DMSO (Sigma Aldrich) and added to aquaria water for 10 days. Tadpoles were maintained in $600 \mathrm{ml}$ of metronidazole solution (maximum 10 tadpoles $/ 600 \mathrm{ml}$ ) at $23.5^{\circ} \mathrm{C}$ in complete darkness (metronidazole is light-sensitive) and changed every $72 \mathrm{~h}$ throughout treatment. For regeneration experiments, metronidazole-treated animals were allowed to recover for up to 8 days in either normal water or containing compounds to be tested at different concentration in ambient laboratory lighting (12h light $12 \mathrm{~h}$ dark).

\section{Quantification of $\mathrm{GFP}^{+}$cells}

GFP was detected directly by fluorescence in live embryos using an AZ100 Nikon Zoom Macroscope. In the optic nerve, the total number of $\mathrm{GFP}^{+}$cells was counted, from the 
emergence of the nerve (i.e., after the chiasm) to the retinal end before and after metronidazole treatment and after treatment with the compound to be tested on the same embryos. Doubleblind counts were performed independently by two researchers. Data were compared to control untreated animals of the same developmental stage. Data presented are the mean \pm SEM of number of GFP+ cells counted on at least 6 tadpoles per condition.

Preparation of sgRNAs, generation of S1P5 null mutant and PCR primers are described in the supplementary materials.

\section{Statistical Analysis}

Prism v5 software was used for statistical analyses. Results are expressed as means \pm SEM. To assess differences between more than two groups when comparing efficiency of remyelinating drugs, parametric two way ANOVA was used (after normality test passed using Shapiro Wilk normality test) followed by an uncorrected Fisher's LSD test. Other $P$-values for comparison of means are from Student's two-tailed $t$-tests. Analysis of covariance was used to analyze regression lines. Statistical significance was set at $p<0.05$. Significant differences are indicated by asterisks: ${ }^{*} p<0.05,{ }^{* *} p<0.01$, and ${ }^{* * *} p<0.001$.

\section{Results}

Oligodendrocyte visualization in MBP-GFP-NTR transgenic Xenopus

In the brain stem, GFP positive cells are detected from stage 43 onward. This corresponds to the developmental stage when myelination markers such as proteolipid protein and MBP are first immunodetected in the hindbrain (stage 42/43) before spreading throughout the brain and spinal cord by stages $46 / 47 .{ }^{9}$ Electron microscope studies on optic nerve ${ }^{10}$ showed myelination to begin in the middle portion at stage $48 / 49$, with numbers of myelinated axons increasing sevenfold between stage 50 and 57 . Distribution of myelin forming oligodendrocytes at stage 48 and 52 is 
shown (Figure 1A-C). Oligodendrocytes populate the ventral half of the hindbrain and spinal cord on each side of the mid-line. At this early developmental stage oligodendrocytes have not yet migrated long distances from their origin and are myelinating the major descending fibre tracts. This is well illustrated by the 3D-reconstruction of whole-mount CNS preparations performed after tissue clarification, i.e. replacement of light-scattering lipids of the brain and spinal cord by a transparent hydrogel matrix (Supplementary file Movie 1).

\section{Conditional ablation and spontaneous repair in the MBP-GFP-NTR Xenopus tadpole}

Stage NF52-53 MBP-GFP-NTR transgenic X. laevis were treated with metronidazole $(10 \mathrm{mM})$ introduced in the aquarium water to induce conditional demyelination. Screening experiments focused on the embryonic optic nerve, easily monitored by live imaging. The number of $\mathrm{GFP}^{+}$ oligodendrocytes is moreover small and very reproducible at each developmental stage, facilitating quantification. After 10 days metronidazole, the number of GFP+ cells per optic nerve significantly decreased from $13.9 \pm 1.0$ to $2.3 \pm 2(\mathrm{p}<0.01)$ (Figure $1 \mathrm{D}$ upper panel). The extent of demyelination was further investigated using electron microscopy (EM) showing the depletion of myelin around axons, and the presence of myelin debris (Supplementary Figure S2 A, B). After 10 days metronidazole exposure, tadpoles were returned to normal water and recovery occurred rapidly: oligodendrocytes per optic nerve reached $8.3 \pm 0.9$ and $13.3 \pm 1.5$ at R3 and R8, respectively (Fig 1D lower panel). Of note, during the 10 days of metronidazole treatment at $23^{\circ} \mathrm{C}$, tadpoles progress from stage 52 to 55 (see $<\underline{\text { http://www.xenbase.org/anatomy/alldev.do }}>$ ); therefore during the recovery period not only demyelinated axons undergo remyelination, but it is likely that in addition some previously naked axons are also myelinated.

Transgenic MBP-GFP-NTR Xenopus tadpoles as a screening platform for molecules enhancing remyelination 
We reasoned that our model could serve for screening potential remyelination-favoring molecules by quantifying remyelination rates as a function of increases in GFP+ cell numbers following demyelination. Since spontaneous repair was often complete at R8, we chose to evaluate repair efficiency only at R3, i.e., 3 days after metronidazole removal. We compared the promyelinating effect of 20 compounds for which an effect on myelination (or remyelination) has been described (Table 1). Among the molecules tested, the three most efficient were, by increasing order: siponimod (3nM), UC-42-WP04 (10nM) and clemastine (100nM)(Figure 2). It is noteworthy that UC-42-WP04, a selective S1PR5 agonist, ${ }^{11}$ was amongst the most efficient remyelinating drugs, while AUY954, a selective S1PR1 agonist, ${ }^{12}$ had no effect. Since siponimod is a dual S1P1 and S1P5 receptor agonist, we investigated further siponimod's remyelinating potential.

\section{Xenopus oligodendrocytes express S1P5 receptor}

As oligodendrocytes in the mouse brain selectively express S1PR $5,{ }^{7}$ we questioned whether S1PR5 expression was also restricted to oligodendrocytes in $X$. laevis. We investigated oligodendroglial expression of S1PR5 in X. laevis by triple immunolabeling of brainstem tissue sections of MBP-GFP-NTR tadpoles (stage 52-53) using a rabbit anti-S1PR5 antibody, a chicken anti-GFP to label mature oligodendrocyte and a goat anti-Sox-10 to label all cells from the oligodendroglial lineage. We observed a perfect cellular co-localisation of Sox10 and S1PR5 (21.5 \pm 6.5 cells $\left./ \mathrm{mm}^{2}\right)$ and all the GFP+ cells were S1P5 $+\left(14.5 \pm 5.3 \mathrm{GFP}^{+} / \mathrm{S} 1 \mathrm{PR} 5^{+}\right.$cells $\left./ \mathrm{mm}^{2}\right)$ Since, in the CNS, Sox10 is an established marker of the oligodendroglial lineage, we concluded that the S1P5+/Sox10+/GFP- cells are most likely OPCs. Altogether our data suggest that in the Xenopus CNS, as in the mouse, S1P5 expression is restricted to the oligodendrocyte lineage and that all myelin-forming oligodendrocytes (GFP+ cells) express S1PR5 (Figure 3). 
To verify that increased numbers of myelinating oligodendrocytes translated into increased myelination, brain stem tissue sections were immunolabeled with anti-MBP, an established myelin marker. Transgenic MBP-GFP-NTR X. laevis ( $\mathrm{n}=18)$ (stage 52-53) were treated for 10 days with metronidazole $(n=15)$, then returned to normal water $(n=3)$ or water containing siponimod $(3 \mathrm{nM})$ for either 3 days $(\mathrm{n}=3)$ or 8 days $(\mathrm{n}=3)$ prior to MBP immunostaining (Figure 4). After 10 days of metronidazole treatment a nearly complete demyelination was achieved (compare D0 and D10 in Figure 4). After metronidazole removal, exposure to siponimod for 3 days (Figure 4 Siponimod R3) resulted in a dramatic increase in myelinated internodes by comparison with spontaneous remyelination (Figure 4 Control R3). At R8 the extent of myelinated fibers was back to the level prior to demyelination (Figure 4 D0, Siponimod R8, and control R8. EM examination of tadpoles following 6 days of recovery confirmed a partial remyelination of MTZ-induced demyelinated axons (Supplementary Figure S2 C), in agreement with the return to nearly normal level of the number of $\mathrm{GFP}^{+}$cells. Evaluation of the g-ratios representing myelin thickness with respect to axon diameter revealed a reduction in myelin thickness, suggestive of remyelination, in siponimod remyelinated animals $(g=0.85 \pm 0.04)$ versus controls not subjected to demyelination $(g=0.82 \pm 0.04, p<0.001)$ (Supplementary Figure S2 D).

\section{The promyelinating effect of siponimod is mediated through S1PR5}

Siponimod is a potent and selective agonist of S1P receptors with an EC50 of 0.39nM for S1P1 and $0.98 \mathrm{nM}$ for S1P5 receptors, respectively. ${ }^{13}$ We questioned which receptor implicated the promyelinating effect of siponimod. As remyelination was observed with the selective S1PR5 agonist (UC-42-WP04), but not with the selective S1PR1 agonist (AUY954) we favored the possibility that siponimod induced remyelination through S1PR5. If this were the case, siponimod should lose efficiency after S1P5 receptor deletion. We took advantage of the clustered regularly interspaced short palindromic repeats (CRISPR)-Cas system (Crispr/Cas9) to test this hypothesis. ${ }^{14}$ To determine the exact mutations of $s 1 p r 5-S$ and $s 1 p r 5-L$ the genomic DNA from 6 
F0 embryos, identified after the T7 endonuclease-based mutation detection, was extracted and sequenced. Each embryo presented different degrees of deletion (between 2 to $169 \mathrm{bp}$ ) leading to shift of the reading frame and premature STOP codon, for both s1pr5-L and s1pr5-S genes (Figure 5A). Each of these embryos at stage 55 was submitted to metronidazole demyelination and treated with siponimod. The pro-remyelinating effect of siponimod was no longer observed in the S1PR5 ${ }^{-/}$embryos (Figure $\left.5 \mathrm{~B}\right)$.

To confirm oligodendroglial S1P5 receptor loss, mutated tadpoles were euthanized after the demyelination-remyelination experiments, and immunolabeled with anti-S1PR5 antibodies on brain stem sections. As illustrated, (Figure 5C), S1PR5 protein was not immuno-detectable in GFP+ cells in any of the six MBP-GFP-NTR; s1pr5 ${ }^{\%}$ tadpoles examined.

\section{Discussion}

For the past 15-20 years significant therapeutic progress has resulted from developing antiinflammatory strategies in MS. These treatments are efficient in diminishing numbers of relapses, but their influence on long-term disability is unclear. Loss of the myelin sheath perturbs normal axon functioning and persistent demyelination renders them vulnerable to irreversible damage leading to axonal transection, with consequent disability accumulation. Spontaneous remyelination can occur, but most often endogenous repair mechanisms are insufficient, notably during aging. The experimental demonstration that environmental clues can restore remyelination potential of aging OPCs indicates that pharmacological approaches may be developed to restore, increase or accelerate remyelination. ${ }^{15}$ Several groups have recently developed in vitro screening approaches for small molecules that drive OPC differentiation. ${ }^{16,17,18}$ These in vitro high throughput screens can identify hit molecule candidates to be further validated in in vivo experimental models of demyelination.

Rodents are the preferred animal models, with primary demyelination induced either by 
detergents to disorganize the myelin sheath (e.g. lysolecithin) or by killing oligodendrocytes with toxins (such as cuprizone or ethidium bromide). In other models demyelination is secondary to an auto-immune attack as in MOG-induced EAE. ${ }^{19}$ Toxin-induced models of demyelination do not mimick MS, but they allow the fundamental biology of remyelination to be explored without the confounding and complicating involvement of an autoimmune process. ${ }^{20}$ Although rodents may be considered as the "standard" experimental model to investigate demyelination and remyelination, they are costly, time consuming and not ideal for longitudinal investigation in the same animal. Although the last decade has seen considerable progress for in vivo imaging of live cells in the normal and demyelinated CNS, ${ }^{21}$ technical issues strongly limit imaging in deep layers of mouse brain, such as myelinated axonal tracts. Tadpole transparency allowed us to overcome these limitations by using transgenic Xenopus expressing fluorescent reporter and a suicide protein in oligodendrocytes.

We present results on 20 molecules previously described, usually in vitro, as candidates to promote remyelination. The Xenopus results are in good agreement with reports on pro-myelinating efficacy of clemastine, ${ }^{17}$ benztropine ${ }^{16}$ and retinoic acid $^{22}$ in mammalian cells. A recent small size phase 2 study of clemastine in chronic optic neuritis showed some positive results. Concerning siponimod no data on remyelination is reported, but a recently completed phase 3 study showed some positive results in secondary progressive MS. ${ }^{5}$ Fingolimod, (acting on S1PR1, S1PR2, S1PR3 and S1PR5, and which is used in the clinic) also promoted remyelination, a finding in agreement with previous data in culture. ${ }^{23}$

In contrast, clobetasol and miconasole, known to be efficient remyelinating candidates in vitro as well as in vivo in the mouse ${ }^{18}$ were without effect in the Xenopus model. One possible explanation is that these molecules were screened for their ability to generate new oligodendrocytes, while in the MBP-GFP-NTR Xenopus model there is no need to generate new OPCs since remyelination results from maturing OPCs (Sox10+ cells) already present in the optic nerve prior to demyelination, without requiring additional production of OPCs. ${ }^{24}$ Generation of new 
oligodendrocytes is only one possible mechanism to favor remyelination. It has been described that OPCs are present in some chronic lesions of MS, so remyelination is not only limited by an absence of precursors or their failure to generate oligodendrocytes, but by the defective maturation of OPCs. ${ }^{25}$

However, an advantage of Xenopus as opposed to other aquatic models, lies in myelin biology and the fundamental difference in the molecular composition of myelin between teleosts and tetrapods. Teleosts, and zebrafish in particular, use $\mathrm{P} 0$ as major myelin protein both in the CNS and the peripheral nervous system (PNS). In contrast, starting with amphibians (including Xenopus), a dramatic evolutionary change in myelin composition between PNS and CNS occurred. In tetrapods (including amphibians and mammals) the major myelin protein in the PNS remains P0, while in the CNS it is PLP and MBP. This event occurred in amphibians and has been maintained throughout evolution up to mammals, including humans.

An advantage of the Xenopus model is read-out rapidity: after 10 days of demyelination, remyelination effects were seen by 3 days recovery (hence a total of 13 days), while the cuprizone model in rodent requires at least 6 weeks for demyelination and an additional 3 weeks for recovery. A final advantage of the Xenopus model is that assessing demyelination and remyelination is performed on the same animals and does not require immunohistochemistry. Although the Xenopus model, as described, is more indicated as a medium throughput screen, it has the potential to be automatized for high-throughput screens. Live imaging of remyelination (automated counting of GFP+ cells) is theoretically possible through the modification of existing automated devices such as the VAST (Vertebrate Automated Screening Technology, Union Biometrica, MA, USA). This device allows the automated pumping from multiwell plates and automated imaging at cellular level of whole zebrafish embryos. This system is limited by the valve and motor pin diameter, which has a maximum of 700 micron diameter. To be 
transposable to Xenopus tadpoles would require adapting the technology by using larger size capillaries. Overall, the procedure is far more efficient when compared with the efforts, time and cost necessary to perform the same type of experiment in mice. However, whilst ideal for screening small molecules, a limit of the MBP-GFP-NTR Xenopus model is that it is not designed to test antibodies, such as anti-Lingo, which would require to be injected into the tadpole and not simply added to the swimming water. Another limitation is that the Xenopus tadpole model may not be adapted to screen drugs aimed at overcoming the decline in remyelination that occurs with advancing age particularly for long-term demyelinating diseases such as MS.

In addition to the rapidity with which the test can be performed, another appreciable advantage of the Xenopus model is using Crispr/Cas9 technology so the molecular mechanism of a given compound can be dissected, with ease and precision. ${ }^{14}$ In the case of siponimod, not only S1P5 receptor was directly deleted in the MBP-GFP-NTR- lineage, but the effect of the deletion was tested on 6 different $\mathrm{KO}$ animals. Three arguments strongly support that the remyelinating potency of siponimod is mediated through the S1P5 receptor, first, another S1PR5 agonist improved remyelination; second, in the absence of S1PR5 siponimod was no longer active and, third, a S1PR1 agonist was inefficient. We did not attempt to delete S1P1 because it is established that within endothelial cells this receptor is required for the coverage of vessels by vascular smooth muscle cells ${ }^{28}$ and lethality occurs in embryos of S1P1 receptor-deficient mice, due to rupture of the unreinforced vessels. ${ }^{27}$ However, although unlikely, we cannot definitively exclude that some of the benefit from siponimod could be from its potential to act through a different mechanism than a S1PR5 agonist.

A recently completed Phase III randomized, controlled study of siponimod in secondary progressive MS has met its primary endpoint, compared with placebo. ${ }^{5}$ Obinata and co-workers have reported the genetic variations of the s1pr1 coding region from exon sequencing of about 12,000 individuals and their functional consequences, suggesting that individual genetic variations 
of s1pr1 can influence receptor function and, therefore, infer differential interaction with S1PR1targeted therapeutics. ${ }^{28}$ Concerning $s 1 p r 5$, deletion of $s 1 p r 5$ has no apparent evident clinical phenotype in either mouse or Xenopus. Whether patients with s1pr5 mutation or deletions may differ in their remyelination capacity, disease severity and/or response to siponimod is an as yet unexplored hypothesis. 


\section{Acknowledgments}

We thank Pierre Affaticati and TEFOR Infrastructure for CLARITY and 3D imaging of tadpoles. AM and BZ are thankful to the Marine Biological Laboratory, National Xenopus Resource for helpful introduction to gene editing technologies in the Xenopus.

\section{Author Contribution}

A.M. and B.Z. conceived the studies and designed the experiments. A.M. performed most of the experiments. S.S. carried out the immunohistochemistry experiments using S1PR5 antibodies. L.A. and Q.V. carried out screening of drugs. J.B.F. carried out the CLARITY experiments. C.G. provided Cas9 protein and advices on Crispr/Cas9 genome editing. D.D., J.L.T., C.L. and B.D. provided technical support and intellectual inputs. B.Z. wrote the paper with inputs from all coauthors.

\section{Declaration of Conflicting Interests}

B.Z. received research support and travel grant from Novartis Pharma, which own patent rights to siponimod (BAF312). B.D. is a founder of the company WatchFrog and D.D. is an employee of WatchFrog. C.L. has participated to advisory boards for Vertex, Roche, Biogen, Novartis, Genzyme.

\section{Funding}

This work was supported by Inserm, CNRS, UPMC, the program "Investissements d'Avenir" ANR-10-IAIHU-06, NeurATRIS, and ANR grant OLGA to B.D. and B.Z. The study was partially funded by grants from Novartis-France and Novartis-Pharma to BZ. 


\section{References}

1. Franklin RJ. Why does remyelination fail in multiple sclerosis? Nat Rev Neurosci 2002;3(9):70514. Review.

2. Miller RH, Fyffe-Maricich SL. Restoring the balance between disease and repair in multiple sclerosis: insights from mouse models. Dis Model Mech 2010 Sep-Oct;3(9-10):535-9. doi: 10.1242/dmm.001958. Review.

3. Kaya F, Mannioui A, Chesneau A, et al. Live imaging of targeted cell ablation in Xenopus: a new model to study demyelination and repair. J Neurosci 2012;32(37):12885-95.

4. Stankoff B, Demerens C, Goujet-Zalc C, et al. Transcription of myelin basic protein promoted by regulatory elements in the proximal 5 ' sequence requires myelinogenesis. Mult Scler 1996;2(3):125-32.

5. Kappos L, Bar-Or A, Cree B et al. Efficacy and safety of siponimod in secondary progressive multiple sclerosis-Results of the placebo controlled, double-blind, Phase III EXPAND study. ECTRIMS 2016, London Sept. $14^{\text {th }}-17^{\text {th }}$ Abstract 2490.

6. Lee MJ, Van Brocklyn JR, Thangada S, et al. Sphingosine-1-phosphate as a ligand for the G protein-coupled receptor EDG-1. Science 1998;279:1552-1555.

7. Jaillard C, Harrison S, Stankoff B, et al. Edg8/S1P5: an oligodendroglial receptor with dual function on process retraction and cell survival. J Neurosci 2005;25(6):1459-69.

8. Nieuwkoop PD, Faber J. Normal Table of Xenopus laevis (Daudin) 1994. New York.

9. Yoshida M. Oligodendrocyte maturation in Xenopus laevis. J Neurosci Res 1997;50:169 -176.

10. Cima C, and Grant P. Development of the optic nerve in Xenopus laevis. II. Gliogenesis, myelination and metamorphic remodelling. J Embryol Exp Morphol. 1982 Dec;72:251-67.

11. Mattes H, Dev KK, Bouhelal R, et al. Design and synthesis of selective and potent orally active S1P5 agonists. Chem Med Chem 2010;5(10):1693-6. doi: 10.1002/cmdc.201000253.

12. Pan S, Mi Y, Pally C, et al. A monoselective sphingosine-1-phosphate receptor-1 agonist prevents allograft rejection in a stringent rat heart transplantation model. Chem. Biol 2006;13:1227-34.

13. Gergely P1, Nuesslein-Hildesheim B, Guerini D, et al. The selective sphingosine 1-phosphate receptor modulator BAF312 redirects lymphocyte distribution and has species-specific effects on heart rate. BrJ Pharmacol 2012;167(5):1035-47.

14. Jinek M, Chylinski K, Fonfara I, et al. A programmable dual-RNA-guided DNA endonuclease in adaptive bacterial immunity. Science 2012 Aug 17;337(6096):816-21. doi: $10.1126 /$ science.1225829.

15. Ruckh JM, Zhao JW, Shadrach JL, et al. Rejuvenation of regeneration in the aging central nervous system. Cell Stem Cell 2012 Jan 6;10(1):96-103. doi: 10.1016/j.stem.2011.11.019.

16. Deshmukh VA, Tardif V, Lyssiotis CA, et al. A regenerative approach to the treatment of multiple sclerosis. Nature 2013;502(7471):327-32. 
17. Mei F, Fancy SP, Shen YA, et al. Micropillar arrays as a high-throughput screening platform for therapeutics in multiple sclerosis. Nat Med 2014 Aug;20(8):954-60. doi: 10.1038/nm.3618.

18. Najm FJ, Madhavan M, Zaremba A, et al. Drug-based modulation of endogenous stem cells promotes functional remyelination in vivo. Nature 2015;522(7555):216-20.

19. Linington $\mathrm{C}$, Bradl M, Lassmann $\mathrm{H}$, et al. Augmentation of demyelination in rat acute allergic encephalomyelitis by circulating mouse monoclonal antibodies directed against a myelin/oligodendrocyte glycoprotein. Am J Pathol 1988;130(3):443-54.

20. Franklin RJ. Regenerative Medicines for Remyelination: From Aspiration to Reality. Cell Stem Cell 2015;16(6):576-7.

21. Fenrich KK, Weber $\mathrm{P}$, Hocine M, et al. Long-term in vivo imaging of normal and pathological mouse spinal cord with subcellular resolution using implanted glass windows. $J$ Physiol 2012;590(16):3665-75.

22. Huang JK, Jarjour AA, Nait Oumesmar B, et al. Retinoid X receptor gamma signaling accelerates CNS remyelination. Nat Neurosci 2011;14(1):45-53.

23. Miron VE, Ludwin SK, Darlington PJ, Jarjour AA, Soliven B, Kennedy TE, Antel JP. Fingolimod (FTY720) enhances remyelination following demyelination of organotypic cerebellar slices. Am J Pathol. 2010 Jun;176(6):2682-94. doi: 10.2353/ajpath.2010.091234.

24. Sekizar S, Mannioui A, Azoyan L, et al. Remyelination by Resident Oligodendrocyte Precursor Cells in a Xenopus laevis Inducible Model of Demyelination. Dev Neurosci 2015;37(3):232-42. doi: 10.1159/000380817.

25. Chang A, Tourtellotte WW, Rudick R, Trapp BD. Premyelinating oligodendrocytes in chronic lesions of multiple sclerosis. N Engl J Med. 2002 Jan 17;346(3):165-73.

26. Allende ML, Yamashita T, Proia RL. G-protein-coupled receptor S1P1 acts within endothelial cells to regulate vascular maturation. Blood 2003;102:3665-3667.

27. Liu Y, Wada R, Yamashita T, et al. Edg-1, the G protein-coupled receptor for sphingosine-1phosphate, is essential for vascular maturation. J Clin Investig 2000;106, 951-961

28. Obinata $\mathrm{H}$, Gutkind $\mathrm{S}$, Stitham J, et al. Individual variation of human $\mathrm{S}_{1} \mathrm{P}_{1}$ coding sequence leads to heterogeneity in receptor function and drug interactions. J Lipid Res 2014 Dec;55(12):2665-75. doi: 10.1194/jlr.P054163.

29. Demerens C, Stankoff B, Zalc B, Lubetzki C. Eliprodil stimulates CNS myelination: new prospects for multiple sclerosis? Neurology 1999;52(2):346-50.

30. Sedel F, Papeix C, Bellanger A, et al. High doses of biotin in chronic progressive multiple sclerosis: a pilot study. Mult Scler Relat Disord. 2015;4(2):159-69.

31. Fayed N, Morales H, Modrego PJ, Muñoz-Mingarro J. White matter proton MR spectroscopy in children with isolated developmental delay: does it mean delayed myelination? Acad Radiol 2006;13(2):229-35.

32. Makoukji J, Belle M, Meffre D, et al. Lithium enhances remyelination of peripheral nerves. Proc Natl Acad Sci U S A 2012;109(10):3973-8 
33. Hennen S, Wang H, Peters L, et al. Decoding signaling and function of the orphan $G$ protein-coupled receptor GPR17 with a small-molecule agonist. Sci Signal 2013;6(298):ra93.

34. Paintlia AS, Mohan S, Singh I. Combinatorial Effect of Metformin and Lovastatin Impedes T-cell Autoimmunity and Neurodegeneration in Experimental Autoimmune Encephalomyelitis. J Clin Cell Immunol 2013;4.

35. Tyler WA, Gangoli N, Gokina P, et al. Activation of the Mammalian Target of Rapamycin (mTOR) is Essential for Oligodendrocyte Differentiation J Neurosci 2009; 29(19): 6367-6378.

36. Rapôso C, Luna RL, Nunes AK, et al. Role of iNOS-NO-cGMP signaling in modulation of inflammatory and myelination processes. Brain Res Bull 2014;104:60-73.

37. O’Sullivan C, Schubart A, Mir AK, Dev KK The dual S1PR1/S1PR5 drug BAF312

(Siponimod) attenuates demyelination in organotypic slice cultures J Neuroinflammation 2016; 13:31

38. Blanchard B, Heurtaux T, Garcia C, et al. Tocopherol derivative TFA-12 promotes myelin repair in experimental models of multiple sclerosis. J Neurosci 2013;33(28):11633-42.

39. Magalon K, Zimmer C, Cayre M, et al. Olesoxime accelerates myelination and promotes repair in models of demyelination. Ann Neurol 2012 Feb;71(2):213-26. doi: 10.1002/ana.22593. 

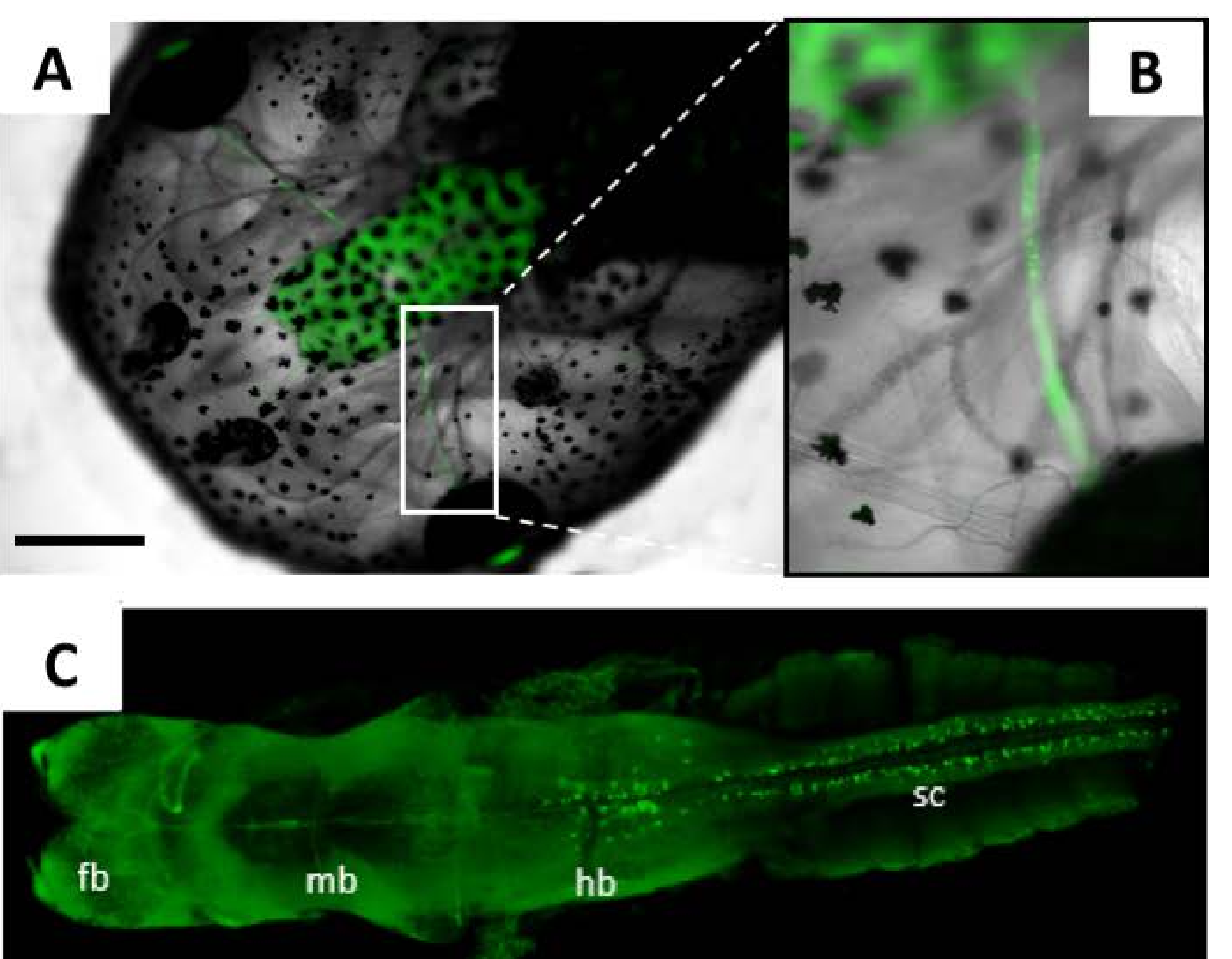

\section{sc}
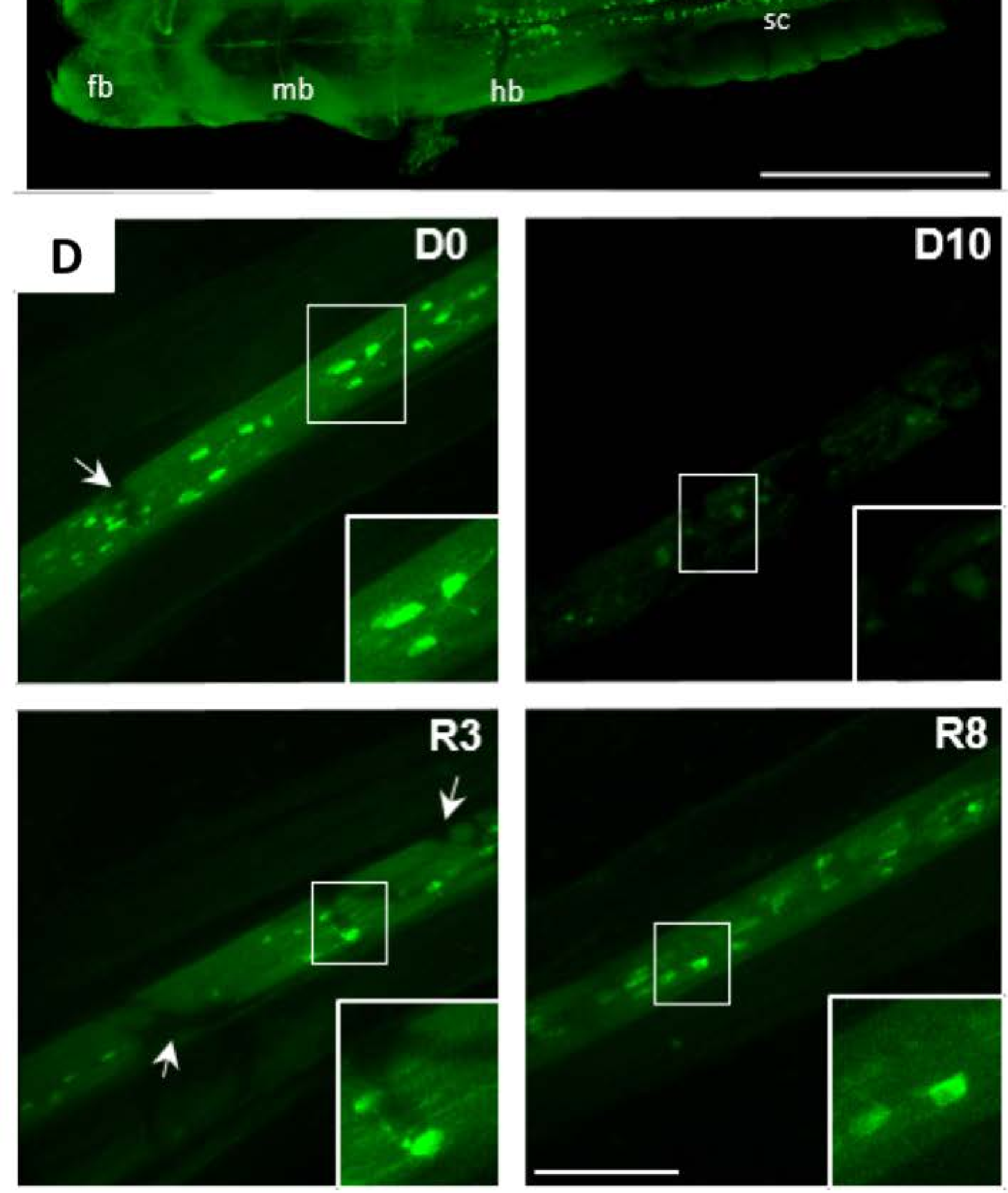


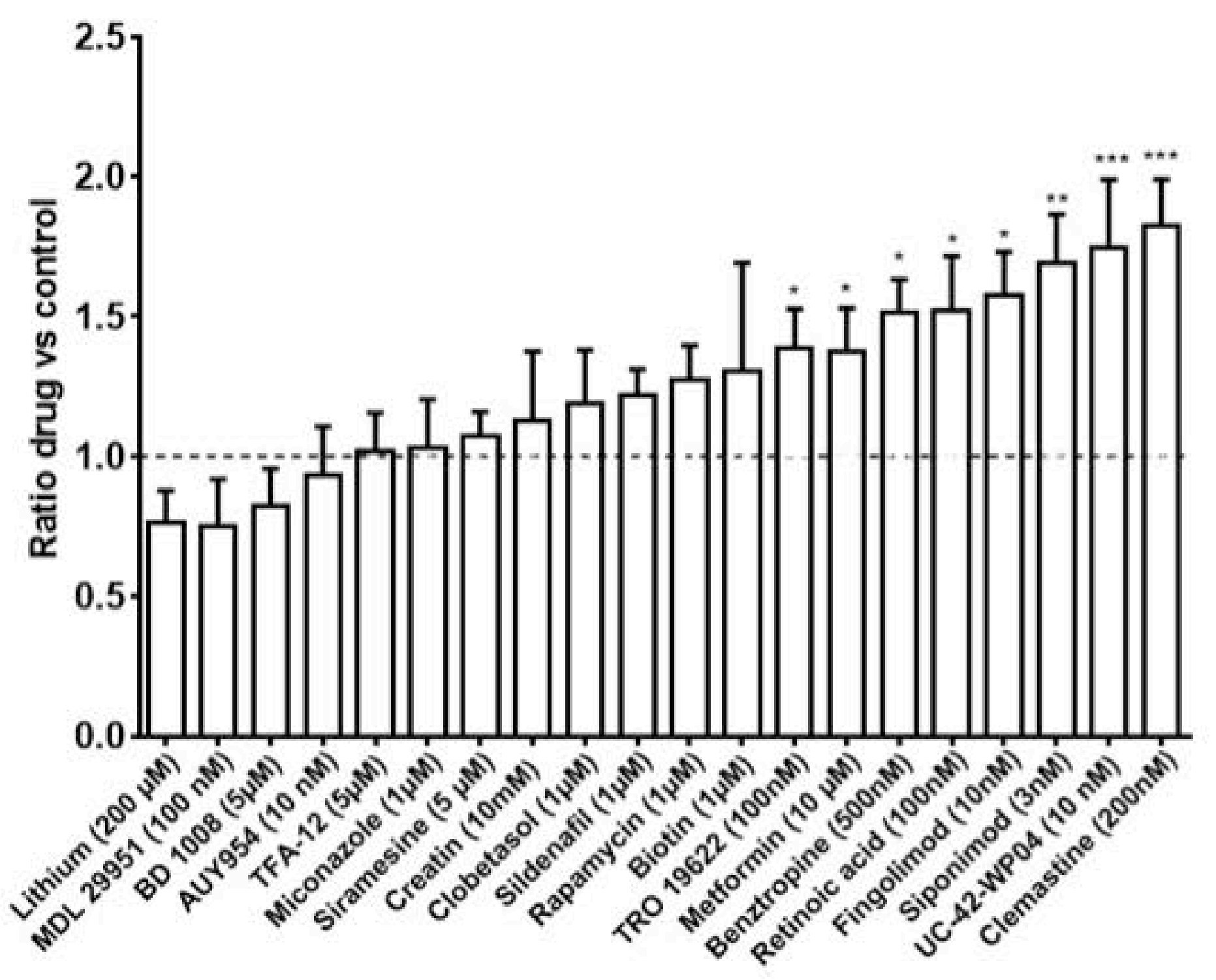


A

B

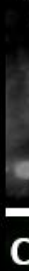

C 


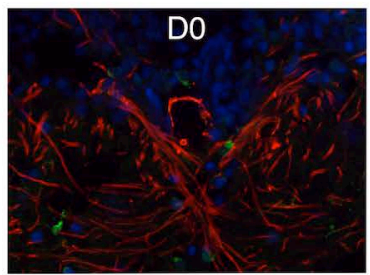

Control R3

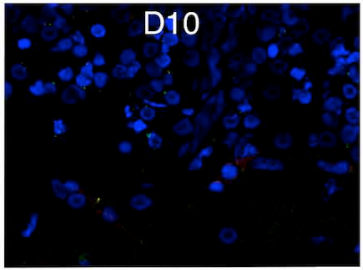

\section{Siponimod R3}

\section{Siponimod R8}

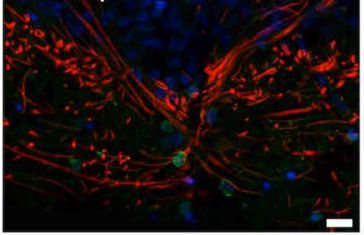


S1P5.L

sgRNA

PAM

ATTCCGATAGACGCCACATTACTATGGTTCGATGAACACTACAGCGGG GACAAGAAGGGCGCATG WT \#1 1 ATTGCGATAGAACGCCACATTACTATGGTCGGATGAAATGA = - -AGCGGGGACAAGAGGGGCGCATG $\triangle 3$-stop(444) \#2ATTGCGATAGAACGCCACATTACTATGGTTCGGATGAAAC - - - - -AGCGGGGACAAGAAGGGGCGCATG $\triangle 5$-stop(677) \#3ATTGCGATAGAACGCCACATTACTATGGTTC-n-n \#4ATTGCGATAGAACGCCACATTAC-n

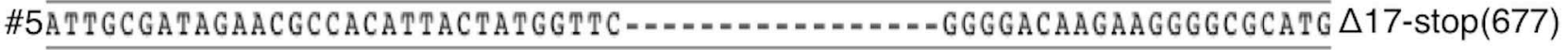
\#6GTTGCGATAGAACGCCACATTACTATGGTCGGAT $=-\cdots=-\cdots=-=-$ GACAAGAAGGGCGCATG $\triangle 16-\operatorname{stop}(514)$

\section{S1P5.S}

sgRNA

PAM

ATTGCGATAGAACG TCACATTACTATGGTTCGATGAAACACTACAGCGGGGACAAGAAGG GTCGATG WT \#1 ATTGCGATAGAACGTCACATTACTATGGTTCGGATGAAAC- -CACAGCGGGGACAAGAAGGTCGCATG $\triangle 2$-stop(710) \#2ATTGCGATAGAACGTCACATTACTATGGTTCGGATGAAA $=-\cdots=--=$-CGGGGACAAGAAGGGCGCATG $\triangle 8$-stop(710) \#3ATTGCGATAGAACGTCACATTACTATGGTTCGGATGAA- - - - - - - - ACAAGAAGGTCGCATG $\triangle 14-\operatorname{stop}(710)$ \#4ATTGCGATAGAACGTCACATTACTA $=-\cdots$ \#5ATTGCGATAGAACG TCACATTACT - \#6ATTGCGATAGAACGTCACATTACT-169-stop(436)
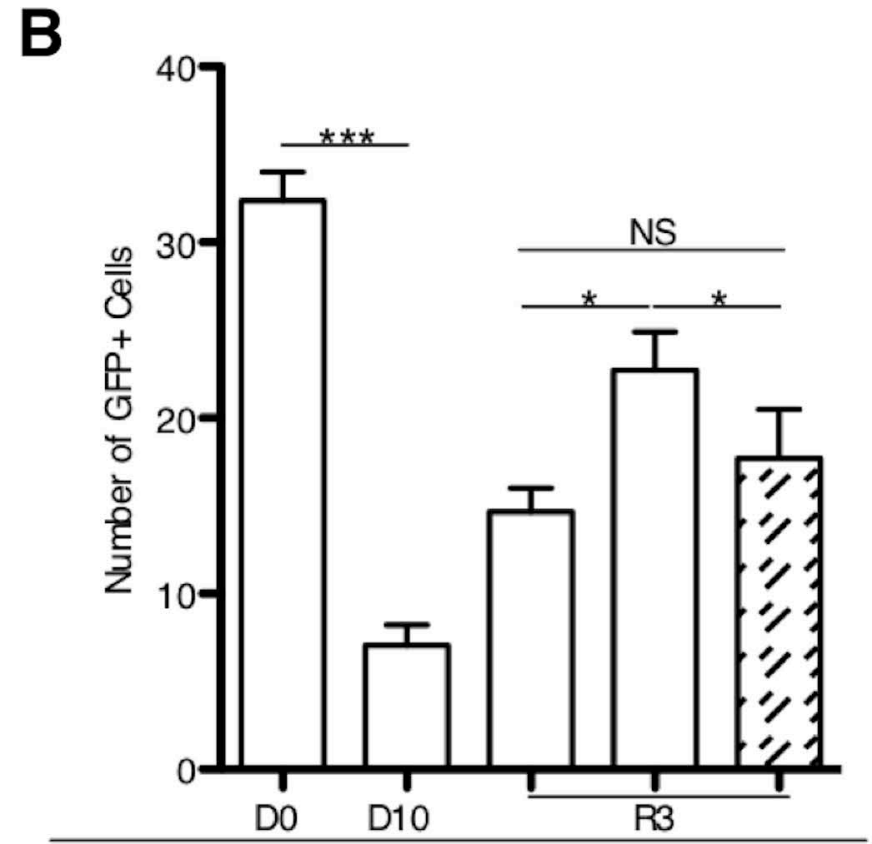

Siponimod
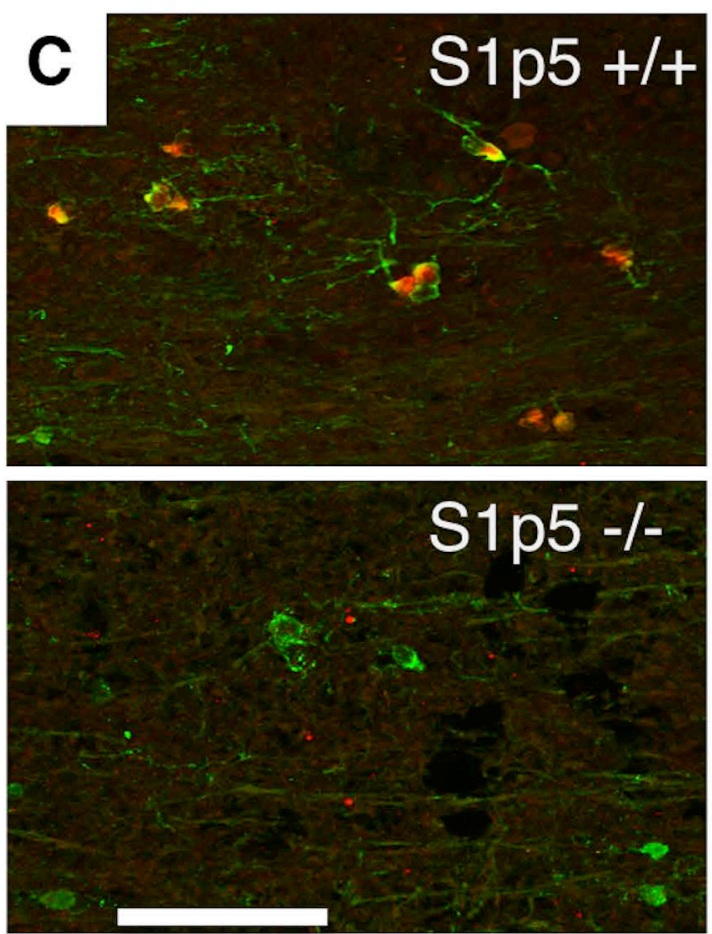
Table 1: List of compounds tested. (a) Concentrations used in vivo (when available) are shown in italics

\begin{tabular}{|c|c|c|c|}
\hline Compound tested & Known mode of action & Concentration used (a) & Reference \\
\hline AUY954 & $\begin{array}{l}\text { Sphingosine } 1 \text { phosphate } \\
\text { receptor } 1\end{array}$ & $1.2 \mathrm{nM} / / 1-10 \mathrm{mg} / \mathrm{kg} / \mathrm{d}$ & (12) Pan et al., 2006 \\
\hline Benztropine & Anticholinergic & $2 \mu \mathrm{M} / / 2.5 \mathrm{mg} / \mathrm{kg}$ & (16) Deshmukh et al., 2013 \\
\hline BD 1008 & Sigma receptor & $5 \mu \mathrm{M}$ & $\begin{array}{l}\text { (29) Demerens et al., } 1999 \\
\text { \& unpublished data }\end{array}$ \\
\hline Biotin & Vitamin B8 & $300 \mathrm{mg} / \mathrm{d}$ & (30) Sedel et al., 2015 \\
\hline Clemastine & $\begin{array}{l}\text { Anti-histaminic anti- } \\
\text { muscarinic }\end{array}$ & $1 \mu \mathrm{M} / 10 \mathrm{mg} / \mathrm{kg}$ & (17) Mei et al., 2014 \\
\hline Clobetasol & Glucocorticoid anti-fungal & $5 \mu \mathrm{M} / / 10 \mathrm{mg} / \mathrm{kg}$ & (18) Najm et al., 2015 \\
\hline Creatine & $\begin{array}{l}\text { Peptide derived from } \\
\text { arginine, glycine \& } \\
\text { methionine }\end{array}$ & Ratio NAA/Cr P<0.16 & (31) Fayed et al., 2006 \\
\hline Fingolimod & $\begin{array}{l}\text { Sphingosine } 1 \text { phosphate } \\
\text { receptor } 1,2,3 \text { and } 5\end{array}$ & $10 \mathrm{nM} / 0.5 \mathrm{mg} / \mathrm{d}$ & (23) Miron et al., 2010 \\
\hline Lithium & Alcalin bipolar disorder & $50 \mathrm{mg} / \mathrm{kg} / \mathrm{d}$ & (32) Makoukji et al., 2012 \\
\hline MDL29951 & NMDA antagonist & $100 \mathrm{nM}$ & (33) Hennen et al., 2013 \\
\hline Metformin & Biguanide Type2 diabetes & $200 \mu \mathrm{M}$ & (34) Paintlia et al., 2013 \\
\hline Miconasole & Imidazole anti-fungal & $1 \mu \mathrm{M} / / 1 \mathrm{mg} / \mathrm{kg}$ & (18) Najm et al., 2015 \\
\hline Rapamycin & Inhibitor of mTOR & $15 \mathrm{nM}$ & (35) Tyler et al., 2014 \\
\hline 9-cis-Retinoic acid & Retinoic receptor & $50 \mathrm{nM}$ & (22) Huang et al., 2011 \\
\hline Sildenafil & Phosphodiesterase inhibitor & $25 \mathrm{mg} / \mathrm{kg} / \mathrm{d}$ & (36) Raposo et al., 2014 \\
\hline Siponimod & $\begin{array}{l}\text { Sphingosine } 1 \text { phosphate } \\
\text { receptor } 1 \text { and } 5\end{array}$ & $1-1000 \mathrm{nM} / / 0.25-10 \mathrm{mg} / \mathrm{d}$ & (37) O’Sullivan et al., 2016 \\
\hline Siramesine & Sigma receptor & $1 \mu \mathrm{M}$ & $\begin{array}{l}\text { (29) Demerens et al., } 1999 \\
\text { \& unpublished data }\end{array}$ \\
\hline TFA-12 & Tocopherol derivative & $0.39 \mathrm{mg} / \mathrm{kg}$ & (38) Blanchard et al., 2013 \\
\hline TRO 19622 (Olesoxime) & Cholesterol derivative & $5 \mathrm{ml} / \mathrm{kg}$ or $60-600 \mu \mathrm{g} / \mathrm{g}$ & (39) Magalon et al., 2012 \\
\hline UC-42-WP04 & $\begin{array}{l}\text { Sphingosine } 1 \text { phosphate } \\
\text { receptor } 5\end{array}$ & $3 \mathrm{nM} / / 3 \mathrm{mg} / \mathrm{kg}$ & (11) Mattes et al., 2010 \\
\hline
\end{tabular}




\section{Supplementary files:}

\section{Supplementary materials:}

\section{CLARITY}

Brains of stage 48 MBP-GFP-NTR tadpoles were submitted to clarification following the procedure developed by TEFOR core facility $<$ http://tefor.net/pages/main/>. Briefly, fixed tadpoles and dissected brains were subjected to clarification following the CLARITY protocol (Chung \& Deisseroth, 2013; Epp et al., 2015) with some tissue-specific adaptations: samples were infused in a cooled solution of extemporaneously prepared hydrogel monomers, $4 \%$ acrylamide and $0.0025 \%$ bis-acrylamide for $2 \mathrm{~d}$ at $4^{\circ} \mathrm{C}$. Hydrogel polymerization was triggered, after degassing, by replacing atmospheric oxygen with nitrogen in a desiccation chamber for $3 \mathrm{~h}$ at $37^{\circ} \mathrm{C}$. Samples were then transferred into embedding cassettes for lipid clearing. The samples were subsequently thoroughly washed in $0.01 \mathrm{M}$ PBS, Tween-20 0.1\%, $2 \mathrm{~d}$ with gentle agitation.

References:

Chung K, Deisseroth K. CLARITY for mapping the nervous system. Nat Methods. 2013 Jun;10(6):508-13. doi: 10.1038/nmeth.2481. Erratum in: Nat Methods. 2013 Oct;10(10):1035.

Epp JR, Niibori Y, Liz Hsiang HL, Mercaldo V, Deisseroth K, Josselyn SA, Frankland PW. Optimization of CLARITY for Clearing Whole-Brain and Other Intact Organs(1,2,3). eNeuro. 2015 May 25;2(3). pii: ENEURO.0022-15.2015. doi: 10.1523/ENEURO.0022-15.2015.

\section{Imaging using a high refractive index solution}

A fructose-based high refractive index solution (fHRI) was prepared and the refractive index of the solution was adjusted to 1.4571 using a refractometer (Kruss). Before imaging the clarified samples were mounted in 1\% low melting point agarose covered with fHRI for imaging. Wholemount brain fluorescence was captured using a Leica TCS SP8 laser scanning confocal microscope equipped with a Leica HC FLUOTAR L 25x/1.00 IMM motCorr objective. 


\section{Preparation of sgRNAs, and generation of S1P5 null mutant: CRISPR sgRNA were}

designed using the «ChopChop » web tool (http://chopchop.cbu.uib.no/) and in vitro transcribed as described by Nakayama et al., 2013. X. laevis is an allotetraploid composed of two separate diploid genomes $(2 \mathrm{n}=36)$. X. laevis has nine pairs of chromosomes named Xla1S and Xla1L through Xla9S and Xla9L where S (short) and L (long) refer to their relative lengths. The recent sequencing of $X$. laevis genome has identified two homologous genes $s 1$ pr $5 S$ and $s 1$ pr $5 \mathrm{~L}$ encoding two forms (S and L) of S1P5 receptors (Fig 1A-B). To delete both S1P5 receptors we designed sgRNAs targeting both the $\mathrm{L}$ and $\mathrm{S}$ homologs, by choosing a sequence common to both receptors. Briefly, the template was prepared using a PCR-based method using as forward primer (60 nt): 5'-TAATACGACTCACTATA (T7 Promoter)-

GGTTCGGATGAAACACTACAG (target sequence)-GTTTTAGAGCTAGAAATAGCAA3'(complimentary overhang with reverse primer). For PCR Phusion polymerase (NEB) was used using stock CRISPR-R primer (80 nt) (5'-

\section{AAAAGCACCGACTCGGTGCCACT'T'T'T'TCAAGT'TGATAACGGACTAGCCT'TAT'T} TTAACT'TGCTAT'T'TCTAGCTCTAAAAC-3') to generate the complete 100 nt sgRNA DNA template. PCR sgRNA were transcribed using T7 MEGAShortscript kit (Ambion) using $8 \mu$ of purified PCR product in $20 \mu \mathrm{l}$ at $37^{\circ} \mathrm{C}$ for $4 \mathrm{~h}$. RNA was purified by precipitation with isopropanol. The sgRNA have been chosen on the ChopChop site $<\underline{\text { https: / / chopchop.rc.fas.harvard.edu }}>$ as having no off-target. Since it was described that offtarget predictors can fail to detect some off-target sites, due to an inappropriate search algorithm (Doench et al, Nat Biotechnol. 2016;34:184-91) we also used the more recently described

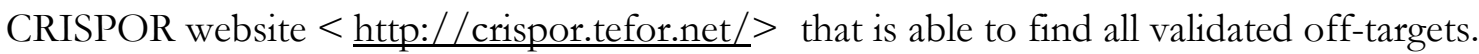
Using this site for GGTTCGGATGAAACACTACAG sgRNA we can find 5 off-targets sites with 3 and 4 mismatches and no mismatches in the 12 bp adjacent to the PAM. However, all these potential off targets are present in introns. We did not confirm these potential sites by sequencing. 
In addition, to facilitate detection of embryos $\mathrm{KO}$ for $s 1 \mathrm{pr} 5$, we co-injected sgRNA for $\operatorname{slc} 45 \mathrm{a} 2$, a gene required for pigmentation, assuming that albinos embryos would also be $\mathrm{KO}$ for $s 1$ pr5 (not shown). sgRNA for slc45a2 were designed by M. Salanga, (National Xenopus Ressource, Woods Hole, MA). Oocytes from MBP-GFP-NTR were fertilized with spermatozoids from wild type male Xenopus laevis and immediately microinjected with a mixture of slc45a2 and s1pr5sgRNA and Cas9 protein. Of note, in preliminary experiments we found that micro-injection in fertilized oocytes of recombinant Cas9 protein was far more efficient compared to Cas9 mRNA to produce total albinism, as evaluated by the degree of mosaic pigmentation of the eyes. To detect s1pr5 mutation among albinos (GFP+) tadpoles, the s1pr5 sequence was PCR amplified and amplicons submitted to T7 endonuclease 1 digestion (Fig 1C).

\section{References:}

Nakayama T, Fish MB, Fisher M, Oomen-Hajagos J, Thomsen GH, Grainger RM. Simple and efficient CRISPR/Cas9-mediated targeted mutagenesis in Xenopus tropicalis. Genesis. $2013 ; 51(12): 835-43$.

Doench JG, Fusi N, Sullender M, Hegde M, Vaimberg EW, Donovan KF, Smith I, Tothova Z, Wilen C, Orchard R, Virgin HW, Listgarten J, Root DE. Optimized sgRNA design to maximize activity and minimize off-target effects of CRISPR-Cas9. Nat Biotechnol. 2016 Feb;34(2):18491. doi: $10.1038 /$ nbt.3437.

\section{PCR primers}

The regions targeted by the complex Cas9 protein-sgRNA were PCR amplified with genomic primers. We used specific forward primers to amplify the $s 1 p 5 \mathrm{~L}$ CTTCTGGCTGGTGTGGCATA and s1p5s (Fn2) CT'TCACCGGGAAGCTCAACA, respectively. Reverse primer (Rd1) GGTCAACAGCCAGAGACTTGGC was common to s1p5L and $s 1 p 5 S$. PCR parameters were: $5 \mathrm{~min}$ at $95^{\circ} \mathrm{C}$ followed by 35 cycles of $95{ }^{\circ} \mathrm{C}$ for $30 \mathrm{~s}$, $72{ }^{\circ} \mathrm{C}$ for $40 \mathrm{~s}$, and $60^{\circ} \mathrm{C}$ for $1 \mathrm{~min}$, with a final $10 \mathrm{~min}$ extension. 


\section{Electron microscopy and morphometry}

Larvae were fixed in a mixture of $2 \%$ paraformaldehyde, $2 \%$ glutaraldehyde, in $0.1 \mathrm{M}$ cacodylate buffer $\mathrm{pH} 7.4$ and $0.002 \%$ calcium chloride overnight at $4^{\circ} \mathrm{C}$, washed in $0.1 \mathrm{M}$ cacodylate buffer, and postfixed in $1 \%$ osmium tetraoxide, $1 \%$ potassium ferricyanide in $0.1 \mathrm{M}$ cacodylate buffer. After washing in cacodylate buffer and water, larvae were incubated in $2 \%$ uranyl acetate aqueous solution at $4^{\circ} \mathrm{C}$ overnight. After rinsing twice in water, larvae were dehydrated in increasing concentrations of ethanol, final dehydratation was in $100 \%$ acetone (twice $10 \mathrm{~min}$ each). Samples were infiltrated with 50\% acetone 50\% Epon for $2 \mathrm{~h}$ and then embedded in Epon (EMBed 812, Electron Microscopy Sciences Cat 14120) Blocks were heated at $56^{\circ} \mathrm{C}$ for $48 \mathrm{~h}$. Ultrathin sections $(70 \mathrm{~nm})$ were examined on an HT7700 electron microscope (Hitachi) operated at $70 \mathrm{kV}$.

Images were analyzed using Image J (NIH) for $g$-ratio measurements by manually drawing 2 lines across the long and short axis of each axon and axon plus myelin (myelinated fiber). Lengths of the lines (in pixels) as generated by ImageJ were averaged across the 2 perpendicular measurements and converted to micrometer $(\mu \mathrm{m})$ using the image scale bars. The $g$-ratio was determined by dividing the mean diameter (long and short axis) of an axon by the mean diameter of the same myelinated fiber. 